\title{
Structure-activity relationship analysis of mosquito glutathione $S$-transferase Noppera-bo and its inhibitors for insecticide development
}

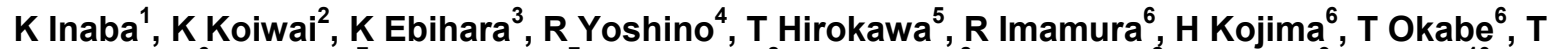

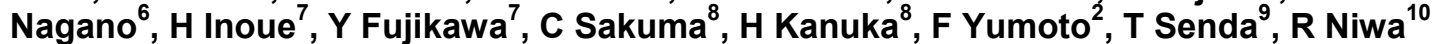 \\ ${ }^{1}$ Grad. Sch. of Life and Environ. Sci, Univ. of Tsukuba, ${ }^{2}$ Structural Biology Research Center, IMSS, \\ KEK, ${ }^{3} \mathrm{Grad}$. Sch. of Life and Environ. Sci, Univ. of Tsukuba, ${ }^{4} \mathrm{Grad}$ Sch Comp Human Sci, Univ. \\ Tsukuba, ${ }^{5}$ Molprof, AIST, ${ }^{6}$ OCDD, Univ. of Tokyo, ${ }^{7}$ Tokyo Univ. of Pharmacol. Life Sci., ${ }^{8}$ Ctr. Med. \\ Entomol., Jikei Univ. Sch. Med., ${ }^{9}$ Structural Bio Research Ctr Inst of Materials Structure , High \\ Energy Accelerator Research Org, Tsukuba, ${ }^{10}$ Life Sci. Center of TARA, Univ. of Tsukuba \\ kze1221@gmail.com
}

[Introduction] A new insecticide targeting mosquitoes is globally needed because mosquitoes are serious vectors of many infectious diseases in the world. In this study, we focused on the insect steroid hormone called ecdysteroid. Ecdysteroid is the principal insect steroid hormone important for regulating developmental transitions such as molting and metamorphosis. Ecdysteroids are biosynthesized from dietary cholesterol through intermediate steps by a series of enzymes. One of the ecdysteroidogenic enzymes, called Noppera-bo (Nobo) (Enya et al. [i]Sci. Rep.[/i] 2014), is a glutathione [i]S[/i] -transferase that catalyzes glutathione conjugation. We focused on Nobo from the yellow fever mosquito [i]Aedes Aegypti[/i] (AeNobo) for insecticide development.

[Results]

1. Identification of small compound inhibitors by high throughput screen

A high-throughput screen of a small molecule library and further validation analysis were conducted to identify compounds that inhibit the AeNobo enzymatic activity. Finally, we found compounds that have a flavonoid nucleus (tentatively designated AeNICs, standing for AeNobo Inhibitor Compounds) as potent inhibitor compounds, which exhibit IC50 values of less than 10 $\mu \mathrm{M}$.

2. Identification of binding mode of the inhibitors with AeNobo by X-ray crystallography

AeNobo was crystallized and soaked with a solution containing AeNIC. AeNobo structure complexed three AeNICs were determined at $1.46 \AA, 1.75 \AA$, and $1.82 \AA$ respectively. All AeNICs bind the hydrophobic site (H-site) of AeNobo (Figure 1).

3. Analysis of protein dynamics by molecular dynamics simulation (MD) ([i]in silico[/i] analysis)

To evaluate the contribution of interaction for binding between AeNICs and AeNobo, MD simulation was conducted. We found that AeNICs consistently interacted with Glu113 of AeNobo (Figure 1).

4. Characterization of structure-activity relationship by in vitro enzyme assay （[i]in vitro[/i] analysis）

4-1 Evaluation of the importance of the hydrogen bond between Glu113 and AeNICs:

A point-mutant protein that substitutes Glu113 with alanine (AeNobo[Glu113Ala]) was constructed and utilized for enzymatic assay. We found that the inhibitory activity of AeNICs was decreased against AeNobo[Glu113Ala] compare to that against wild type AeNobo.

4-2 Evaluation of the importance of planar structure:

Since AeNICs tend to have a planar chemical structure, we hypothesized that a planar structure of AeNICs might affect inhibitory activity. To test the hypothesis, we compared the inhibitory activity of AeNIC derivative compounds with non-planar structures. We found that the IC50 of such derivatives was more than $25 \mu \mathrm{M}$, suggesting the planar structure is essential for inhibitory activity (Table1).

5. mosquitoes larvicidal assay. ([i]in vivo[/i] analysis)

To evaluate whether the AeNIC affects mosquito ([i]Aedes aegypti[/i]) development, a larvicidal assay was conducted. Mosquito larvae were reared into water containing $10 \mathrm{ppm}$ AeNIC and observed their development (Table1). Consistent with in vitro assay, $45 \%$ of AeNIC treated mosquito larvae died. In contrast, non-inhibitors such as AeNIC derivative compounds with non-planar structures did not affect mosquito development (Table1).

[Conclusion] We found two characteristics of AeNIC inhibitory activity against AeNobo. (1) Interaction with Glu113 via a hydrogen bond and (2) the planar structure is essential for inhibitory activity (Table1). Moreover, AeNICs have an insecticidal effect. We will try the identification of essential amino acid residue for the restriction of binding with AeNobo.

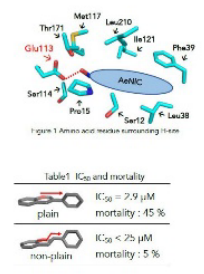

Figure 1.

Acta Cryst. (2020). A76, a173 